\title{
Sex Differences in the Behavioral Effects of 24-h/day Access to Cocaine under a Discrete Trial Procedure
}

\author{
Wendy J Lynch*,' and Jane R Taylor' \\ 'Department of Psychiatry, Yale University, New Haven, CT, USA
}

\begin{abstract}
Although more men than women are addicted to cocaine, it has been suggested that women may have an accelerated transition to addiction, and that once addicted they may be more vulnerable to relapse. Here we investigate the effects of extended access to cocaine under a 24-h/day discrete trial procedure on patterns of intake and subsequent motivation to use cocaine as assessed by responding under a progressive-ratio schedule in male and female rats. Rats were initially trained to self-administer cocaine ( $1.5 \mathrm{mg} / \mathrm{kg} / \mathrm{infusion}) \mathrm{under}$ a fixed-ratio I schedule until acquisition occurred, and then responding was assessed under a progressive schedule for three sessions. Subsequently, rats had 24-h access to intravenous cocaine infusions ( $1.5 \mathrm{mg} / \mathrm{kg})$ that were available in discrete trials $(4,10 \mathrm{~min}$ trials/h) for 7 consecutive days. At 10 days after the last discrete trial session, responding was reassessed under a progressive-ratio schedule for three additional sessions to investigate changes in motivation to obtain cocaine. Prior to cocaine self-administration under the 24-h access discrete trial procedure, males and females did not differ on cocaine self-administration under the fixed-ratio or progressive-ratio schedules. However, sex differences emerged under the 24-h access discrete trial procedure with females self-administering higher levels of cocaine, for longer initial periods of time, and showing a greater disruption in the diurnal control over intake than did males. Additionally, following a 10-day forced abstinence period, females responded at higher levels under the progressive-ratio schedule to obtain cocaine infusions than did males. These findings suggest that extended access to cocaine under the discrete trial cocaine selfadministration procedure produces sex-dependent patterns of intake and sex-specific changes in motivation to obtain cocaine as measured by progressive-ratio responding.

Neuropsychopharmacology (2004) 29, 943-95I, advance online publication, I I February 2004; doi: I 0. I 038/s..npp. I 300389
\end{abstract}

Keywords: addiction; animal model; cocaine; motivation; rat; self-administration; sex differences

\section{INTRODUCTION}

Cocaine addiction has been characterized by a shift from casual, recreational use, to compulsive and binge patterns of use, presumably due to the development of tolerance and/or changes in motivation to use the drug over time (Gawin, 1991; Koob, 1992; Robinson and Berridge, 2001). Although more men than women abuse cocaine (Substance Abuse and Mental Health Services Administration, 2001), women may be at a greater risk on some aspects of cocaine addiction. For example, retrospective reports reveal that women enter treatment programs after fewer years of cocaine use and they take less time to meet criteria for dependence after initial use compared to men, suggesting that in women there is an accelerated transition to addiction

\footnotetext{
*Correspondence: Dr Wendy J Lynch, Department of Psychiatry, Yale University School of Medicine, 34 Park St., New Haven, CT 06516, USA, Tel: + I 203974 7755, Fax: + I 2039747897 ,

E-mail: wendy.lynch@yale.edu

Received 05 December 2003; revised 12 December 2003; accepted 21 December 2003

Online Publication: 23 December 2003 at http://www.acnp.org/ citations/Npp $12230303354 /$ default.pdf
}

(Brady and Randall, 1999; Kosten et al, 1996). Although cocaine-addicted women and men typically report similar levels of cocaine use (Evans et al, 1999; Kosten et al, 1996), women report higher levels of craving following exposure to cocaine-related cues (Robbins et al, 1999), suggesting that during abstinence, women may be more motivated than men to use cocaine (Elman et al, 2001). These differences may be due to sociocultural factors and/or to biological factors. Animal models that mimic different aspects of addiction may be useful in exploring sex differences in biological vulnerability to cocaine addiction.

There has been a recent emphasis on developing animal cocaine self-administration procedures that model the transitional process from use/abuse to addiction. Several new models have been developed (eg Ahmed and Koob, 1998, 1999; Tornatzky and Miczek, 2000; Roberts et al, 2002), and they may be useful in addressing the question of sex differences. Results from studies with male rats have revealed that access to cocaine is critical in producing a transition from controlled, regulated intake, to uncontrolled and dysregulated patterns of intake. For example, Tornatzky and Miczek (2000) examined the effects of unlimited access to cocaine under a fixed-ratio 1 schedule (ie each 
response produced an infusion of cocaine), and found that rats self-administered cocaine in 'binge' patterns that were characterized by a progressive disruption in the regularity of responding over time. However, because of toxicity, these studies had to be truncated to only a few days of cocaine self-administration.

Another method that allows for extended access to cocaine is a discrete trial procedure wherein animals are given 24-h access to cocaine infusions that are available in discrete $10 \mathrm{~min}$ trials (Fitch and Roberts, 1993; Freeman et al, 2002; Lynch and Roberts, 2004; Morgan et al, 2002; Roberts et al, 2002). With this method, cocaine toxicity is limited and self-administration behavior can be maintained for extended periods of time. Under low access conditions (1-2 discrete trials $/ \mathrm{h}, 1.5 \mathrm{mg} / \mathrm{kg} /$ infusion), the behavior of male rats is highly regulated by the light/dark cycle, and responding for cocaine occurs predominately during the dark phase (Lynch and Roberts, 2004; Fitch and Roberts, 1993; Roberts et al, 2002). However, under high cocaine access conditions (ie 4 or 5 discrete trials $/ \mathrm{h}, 1.5 \mathrm{mg} / \mathrm{kg} /$ infusion), the diurnal control over behavior decreases and male rats self-administer cocaine in 'binge/abstinent' patterns taking nearly every infusion available for the first 1-2 days, followed by periods of self-imposed drug abstinence that are interspersed with periods of active drug use (Lynch and Roberts, 2004; Fitch and Roberts, 1993; Roberts et al, 2002; Morgan et al, 2002). An extensive parametric analysis has revealed that as access to cocaine increases, levels of intake increase and become dysregulated and the diurnal control over self-administration behavior decreases (Roberts et al, 2002).

In this study, we were interested in comparing males and females on patterns and levels of intake using 24-h access to cocaine under the discrete trial procedure. While sex differences have not yet been investigated using animal models of the transition from controlled, regulated intake to uncontrolled and dysregulated patterns of intake, sex differences have been reported during other phases of the addiction process (for reviews see Lynch et al, 2002; Mello and Mendelson, 2002). For example, sex differences have been observed during initiation, or acquisition, of cocaine self-administration. Specifically, under low-dose conditions, female rats acquire cocaine self-administration at a faster rate than do male rats (Lynch and Carroll, 1999; Carroll et al, 2002; Hu et al, 2004). Under maintenance, or steady-state conditions, like humans, male and female animals typically do not differ in levels of cocaine intake when responding is assessed under a simple fixed-ratio schedule (Lynch and Carroll, 1999; Roberts et al, 1989). However, when more challenging behavioral schedules are used sex differences have been reported. For example, when responding for low doses of cocaine is assessed under a progressive-ratio schedule, in which the ratio requirement to obtain a cocaine infusion progressively increases within a session, female rats reach much higher final ratios suggesting that females are more motivated to obtain cocaine than are males (Carroll et al, 2002; Roberts et al, 1989). Similar results have been observed under relapse, or reinstatement, testing conditions (Lynch and Carroll, 2000). Critically, in all of the above studies, animals were tested under conditions that resulted in controlled and regular patterns of drug intake (eg 1-3 h sessions/day; low doses of drug). The question remains as to whether sex differences are relevant under conditions that are more analogous to human cocaine abuse, that is under conditions that promote uncontrolled and dysregulated patterns of intake (Gawin, 1991). Thus, the present experiment compared male and female rats on responding for cocaine using cocaine access conditions that have been shown previously in male rats to promote high levels of intake and dysregulated patterns of self-administration (ie 4 discrete trials $/ \mathrm{h}, 1.5 \mathrm{mg} / \mathrm{kg} /$ infusions of cocaine; Lynch and Roberts, 2004; Fitch and Roberts, 1993; Roberts et al, 2002; Morgan et al, 2002).

A second goal of this study was to characterize changes in motivation to obtain cocaine infusions following cocaine self-administration under high access conditions. In humans, motivation to use drugs is presumed to change over time, and it has been suggested that increases in motivation and/or the development of tolerance may serve as a trigger for relapse and to maintain the addiction cycle (Gawin, 1991; Jentsch and Taylor, 1999). Recent work with male rats has revealed that cue-induced reinstatement responding (Grimm et al, 2001) and motivation to use cocaine (as measured by progressive ratio responding; Morgan et al, 2002) increases over a cocaine abstinence period, and may reflect the development of sensitization to the reinforcing effects of cocaine (Robinson and Berridge, 2001). In order to determine whether there are sex differences in motivation to use drug following cocaine self-administration under high access conditions, animals were retested on responding for cocaine under a progressive-ratio schedule after a 10-day forced abstinence period.

\section{METHODS}

\section{Subjects}

Age-matched, sexually mature female $(n=20)$ and male $(n=18)$ Sprague-Dawley rats between 90 and 100 days and weighing approximately $280-310 \mathrm{~g}$ (female) and $380-410 \mathrm{~g}$ (male) at the start of the study were used as subjects. Upon arrival at the facility, and throughout the experiment, animals were maintained on a 12-h light/dark cycle (lights on at 0700) with free access to food and water. After a 5-day acclimation period, rats were anesthetized with equithesin $(4.32 \mathrm{mg} / \mathrm{kg})$ and implanted with a chronic indwelling cannula into the right jugular vein using methods that have been described previously (Lynch and Carroll, 2000). After cannulation, the rats were placed in individual operant chambers (ENV-018M; Med Associates, St Albans, VT), and once grooming and eating behaviors resumed, behavioral testing began (typically after $24 \mathrm{~h}$ of recovery). Rats were weighed a minimum of once per week, and immediately prior to and following $24 \mathrm{~h}$ access to cocaine under the discrete trial procedure. In order to control for the stress of handling, animals were not weighed during the 7-day period in which cocaine was available under the 24-h/day discrete trial procedure. Cannula patency was assessed approximately every 7 days, and if a cannula was not patent a new one was implanted into the left jugular vein and testing resumed a minimum of $24 \mathrm{~h}$ later. The health of the animals was monitored daily. The experimental protocol was 
approved by the Animal Care and Use Committee of Yale University and was conducted in accordance with guidelines set by the National Institutes of Health.

\section{Experimental Procedures}

Cocaine self-administration training under a fixed-ratio schedule. In order to ensure that all animals acquired cocaine self-administration, rats were initially trained to self-administer a high dose of cocaine under a fixed-ratio 1 schedule of reinforcement. Specifically, after a minimum of 1 day recovery from surgery, the rats were allowed to selfadminister cocaine infusions $(1.5 \mathrm{mg} / \mathrm{kg})$ during daily sessions beginning at 1200. At the beginning of each training session, the left lever (cocaine-associated lever) was extended into operant chamber and each response on it produced an infusion of cocaine, and the stimulus light about the lever was illuminated for the duration of the infusion (approximately $5 \mathrm{~s}$ ). Each rat received one 'priming' infusion at the beginning of each training session. The left lever remained extended into the chamber and cocaine infusions were available until a total of 20 injections were obtained. Rats generally began self-administering cocaine at the start of each daily session (1200) and obtained all 20 infusions in approximately $3 \mathrm{~h}$ (1500). The right lever (activity lever) was extended into the chamber for the duration of the experiment, and responses on it were recorded but produced no consequence. Responding was assessed daily under the fixed-ratio schedule until rats met criteria for acquisition. Acquisition of cocaine self-administration was defined as five consecutive sessions in which rats earned all 20 infusions that were available.

Cocaine self-administration under a progressive-ratio schedule. Once rats acquired cocaine self-administration under a fixed-ratio schedule, responding was assessed under a progressive-ratio schedule to determine whether males and females differed in motivation to obtain cocaine infusions $(1.5 \mathrm{mg} / \mathrm{kg})$ prior to daily 24 -h access to cocaine under the discrete trial procedure. At the beginning of each progressive-ratio session, the left lever (cocaine-associated lever) was extended into operant chamber and remained extended until $10 \mathrm{~min}$ before the next progressive-ratio session. Each rat received one 'priming' infusion at the beginning of each training session. The right lever (activity lever) was extended into the chamber for the duration of the experiment, and responses on it were recorded but produced no consequence. Responding on the left lever was reinforced under a progressive-ratio schedule. With this procedure, the response requirement to obtain a cocaine infusion increased progressively throughout the session in the following steps: $1,2,4,6,9,12,15,20,25,32$, $40,50,62,77,95,118,145,178,219,268,328,402,492,603$, etc (as described in Arnold and Roberts, 1997) until rats no longer respond. Although the lever was available for almost $24 \mathrm{~h}$ (sessions started at 1200 and ended at 2350), responding typically ceased within $3 \mathrm{~h}$. Responding was assessed for three consecutive sessions in order to determine baseline breakpoints (defined as the number of infusions self-administered in a session).
Cocaine self-administration under a 24-h access discrete trial procedure. Following the third progressive-ratio session, responding was assessed for seven consecutive sessions under a 24 -h discrete trial procedure to determine whether males $(n=18)$ and females $(n=20)$ differed on patterns and levels of intake under extended cocaine access conditions. However, over the course of the 7 days 24-h access discrete trial procedure, signs of toxicity (eg rapid weight loss, death) were apparent in three out of the 20 females rats tested and these animals were removed from the study and their data were not included in the final analyses. Trials were initiated every 15-min for a total of 7 days. Each 10-min trial began with the introduction of the left lever into the chamber. Each response on this lever during a 10 -min trial produced an infusion of cocaine ( $1.5 \mathrm{mg} / \mathrm{kg} /$ infusion), and the stimulus light above the lever was illuminated for the duration of the infusion (approximately $5 \mathrm{~s}$ ). A discrete trial was terminated and the lever was retracted following a response under a fixed-ratio 1 schedule or after $10 \mathrm{~min}$ had elapsed.

Progressive-ratio responding following cocaine selfadministration under a $24-h$ access discrete trial procedure. In order to examine sex differences in motivation to obtain cocaine following extended access to cocaine a subset of the male $(N=12)$ and female $(N=8)$ rats were retested on responding for cocaine under the progressive-ratio schedule. Following the last 24 -h discrete trial session under the discrete trial procedure, rats were removed from the operant testing chambers and individually housed in a colony room in order to make more efficient use of the operant testing chambers. Rats remained in these boxes for 10 days, and catheters were flushed daily with $0.2 \mathrm{~cm}^{3}$ of heparinized saline $\left(0.2 \mathrm{~cm}^{3}\right.$ of $\left.30 \mathrm{U} / \mathrm{ml}\right)$. At 10 days after the last discrete trial cocaine self-administration session, rats were weighed, rehoused in operant test chambers, and cocaine self-administration behavior was reassessed. This time-point was selected based on previous findings with male rats showing that 7-10 days of extended access to cocaine self-administration under the discrete trial procedure (5 trials $/ \mathrm{h}, 1.5 \mathrm{mg} / \mathrm{kg} /$ infusion), coupled with a forced abstinence period of at least 7 days produced increases in progressive-ratio responding (Morgan et al, 2002). Importantly, previous work has shown that progressive-ratio responding is incredibly stable over time, and that following either low cocaine access conditions and/or immediately following high access conditions, progressive-ratio responding either does not change or is decreased (Morgan et al, 2002; Roberts et al, 2002). During the first retest session, cocaine infusions $(1.5 \mathrm{mg} / \mathrm{kg})$ were available under a fixedratio 1 schedule (20 maximum) to ensure cannula patency. Subsequently, responding was reassessed under the progressive-ratio schedule for three consecutive sessions under identical conditions as those used initially (see above). Although the lever was available for almost $24 \mathrm{~h}$ (sessions started at 1200 and ended at 2350), responding typically ceased within $5 \mathrm{~h}$.

Other animals were either not retested due to problems with cannula patency ( $n=1$ female), signs of toxicity during withdrawal from the 24 -h access discrete trial procedure (eg rapid weight loss, ceasing of feeding and grooming 
behaviors; $n=1$ female), or were used for other behavioral or molecular studies (data not shown). In order to determine whether the estrous cycle was functioning in females self-administering high doses of cocaine, several of the rats were vaginal swabbed and estrous cycle phase was determined. Although this was done randomly in only a few rats, and the data are not presented, results indicate that the rats were cycling. The testing procedure did not interfere with the experimental protocol.

\section{Data Analysis}

The mean number of days to acquire cocaine selfadministration were compared between males $(n=18)$ and females $(n=17)$ using an unpaired $t$-test. Interdose intervals were determined for the last 3 of the 5 days of cocaine acquisition under the fixed-ratio schedule and an unpaired $t$-test was used to compare the overall means between males and females. Final ratios reached under the progressive schedule were determined for each of the three test sessions, and the mean number of infusions obtained for each animal these were compared between males $(n=18)$ and females $(n=17)$ using an unpaired $t$-test.

During sessions in which cocaine was available under the 24-h discrete trial procedure, a record of reinforced and nonreinforced trials was kept for each subject for each of the 7 days (ie there were 96 trials/day and a record of whether or not an infusion was obtained for each trial was recorded and analyzed sequentially over the 7-day testing period). Under this procedure, males $(n=18)$ and females $(n=17)$ were compared on mean daily cocaine intake, initial 'binge' length, and diurnal patterns of responding. Initial 'binge' length was defined as a series of at least four infusions with no periods of cocaine abstinence greater than $1 \mathrm{~h}$. This definition was based on previous observations from male rats that had 24-h access to cocaine under the discrete trial procedure (Roberts et al, 2002). Specifically, analyses of individual records from male rats self-administering cocaine under the discrete trial procedure revealed that $1 \mathrm{~h}$ of abstinence typically marked the end of a period of active drug-taking behavior and the beginning of an extended abstinence period. Initial 'binge' length and total intake over the 7 days were compared between males and females using separate unpaired $t$-tests. The trial by trial data were converted to hourly intake. Total intake during the light phase and the dark phase were obtained for both males and females and averaged over days 1 and 2 and days 3-7 and subjected to repeated measures ANOVA with phase as the repeated measures and sex as the betweengroup measure.

The final ratios reached at baseline (prior to discrete trial self-administration) and at retest were obtained for males $(n=12)$ and females $(n=8)$ and the mean number of infusions self-administered were compared using repeated measures ANOVA with time as the within-subject factor and sex as the between-subject factor. The change from baseline was assessed within males and females using a paired $t$-test. Statistical analyses were conducted using SPSS 10 , and findings were considered statistically significant if $p$-values were less than 0.05 .

\section{Drugs}

Cocaine hydrochloride was purchased from Sigma Chemical Company (St Louis, MO), and dosages are expressed as the salt. Cocaine was mixed in sterile $0.9 \%$ saline and passed through a microfilter. Cocaine infusions $(1.5 \mathrm{mg} / \mathrm{kg})$ were delivered at a rate of $0.024 \mathrm{ml} / \mathrm{s}$ and the infusion duration was adjusted according to the body weight of each subject $(1.5 \mathrm{~s} / 100 \mathrm{~g})$. A relatively high dose of cocaine was tested in these experiments $(1.5 \mathrm{mg} / \mathrm{kg})$. This dose was selected based on previous work showing that low doses of cocaine (0.2$0.6 \mathrm{mg} / \mathrm{kg}$ ) maintain higher levels of responding in females compared to males when responding was assessed under the fixed-ratio (ie Lynch and Carroll, 1999) and the progressive-ratio schedule (ie Carroll et al, 2002), and on pilot studies in our laboratory showing that males and females respond at similar levels to obtain $1.5 \mathrm{mg} / \mathrm{kg}$ infusions of cocaine under both of these schedules. Additionally, in previous studies with male rats selfadministering cocaine under a 24 -h discrete trial procedure, this dose of cocaine produced high levels of intake and dysregulated patterns of use (Roberts et al, 2002).

\section{RESULTS}

\section{Cocaine Self-Administration under Fixed-Ratio and Progressive-Ratio Schedules}

Table 1 shows data for acquisition and maintenance of cocaine self-administration under the fixed-ratio 1 schedule when a maximum of 20 infusions were available and number of infusions obtained under the progressive-ratio schedule prior to cocaine self-administration under the $24-\mathrm{h}$ access discrete trial procedure. Males and females did not differ in rates of acquisition of cocaine self-administration or in rates of responding for cocaine during the last three sessions under the fixed-ratio 1 schedule $(p>0.05)$. Additionally, males and females obtained similar numbers of infusions under the progressive-ratio schedule $(p>0.05)$ indicating that males and females were equally motivated to

Table I Summary of Cocaine Self-Administration Behavior Measured under the Fixed-Ratio, the Progressive-Ratio, and Discrete Trial Schedules for Female $(n=17)$ and Male $(n=18)$ Rats

\begin{tabular}{|c|c|c|c|c|}
\hline Schedule & Measure & Female & Male & $P$-value \\
\hline \multirow[t]{2}{*}{ Fixed-ratio } & $\begin{array}{l}\text { Days to } \\
\text { acquire }\end{array}$ & $8.5( \pm 0.7)$ & $9.9( \pm 0.8)$ & $>0.1$ \\
\hline & $\begin{array}{l}\text { Mean } \\
\text { interdose } \\
\text { interval (min) }\end{array}$ & $9.7( \pm 0.4)$ & $9.6( \pm 0.5)$ & $>0.1$ \\
\hline $\begin{array}{l}\text { Progressive- } \\
\text { ratio }\end{array}$ & $\begin{array}{l}\text { Number of } \\
\text { infusions }\end{array}$ & |4.| $( \pm 0.8)$ & $14.0( \pm 0.8)$ & $>0.1$ \\
\hline \multirow[t]{2}{*}{$\begin{array}{l}\text { Discrete trial } \\
\text { (24-h access) }\end{array}$} & $\begin{array}{l}\text { Initial 'binge' } \\
\text { length }(h)\end{array}$ & $39.2( \pm 3.8)$ & $28.7( \pm 3.3)$ & $<0.05^{*}$ \\
\hline & $\begin{array}{l}\text { Mean daily } \\
\text { intake (mg/kg) }\end{array}$ & $103.7( \pm 4.0)$ & $88.8( \pm 3.0)$ & $<0.05^{*}$ \\
\hline
\end{tabular}

*Significantly different by unpaired t-test. 
obtain infusions of cocaine. These results indicate that prior to cocaine self-administration under the 24-h access discrete trial procedure, the dose of cocaine tested $(1.5 \mathrm{mg} / \mathrm{kg} /$ infusion) maintained similar levels of responding in males and females under both the fixed- and progressive-ratio schedules.

\section{Cocaine Self-Administration under a 24-h Access Discrete Trial Procedure}

Table 1 shows data for mean daily cocaine intake and initial 'binge' length observed under the 24-h access trial procedure for male and female rats. Sex differences emerged under the discrete trial procedure with females selfadministering more cocaine than males over the 7 days $(t=2.4, \mathrm{df}=33, p<0.05)$, and showing a longer initial 'binge' length (defined as a series of at least four cocaine infusions with no periods of cocaine abstinence greater $1 \mathrm{~h}$ ) $(t=2.1, \mathrm{df}=33, p<0.05)$ compared to males.

Females and males also differed in their diurnal patterns of cocaine self-administration over the 7 days (Figure 1), and this sex differences appears to be accounted for during the last five sessions under the 24-h access discrete trial procedure. Specifically, during the first 2 days under this procedure (Figure 1a), patterns of intake in males and females were similar and they were characterized by maximal levels throughout the dark phase (1900 to 0700) that peaked within the early light phase (0700 to 1300) followed by a gradual decrease to submaximal levels during the late light phase (1300 to 0700). Averaged across the first 2 days of 24 -h access under the discrete trial procedure, the percent of total responding that occurred in the dark phase was $51 \%( \pm 2)$ for females and $54 \%( \pm 2)$ for males. A repeated measures ANOVA comparing females and males on levels of cocaine intake averaged across days 1 and 2 as a function of phase (light $v s$ dark) revealed a significant main effect of phase $(\mathrm{F}(1,33)=12.9, p<0.01)$, but a nonsignificant effect of sex $(p>0.05)$, a nonsignificant interaction of sex and phase $(p>0.05)$.

After the initial 2 days under the 24 -h access discrete trial procedure (Figure 1b), sex differences were apparent in patterns of intake. For females, during the last 5 days under the 24-h access discrete trial procedure intake was characterized by progressively increasing levels of intake throughout the dark phase (1900 to 0700) that peaked within the early light phase (0700 to 1300 ) followed by a gradual decrease to submaximal levels during the late light phase (1300 to 1900). For males, responding was more selective to the dark phase and patterns were characterized by progressively increasing levels of intake within the dark phase (1900 to 0700) that peaked within the late dark phase (0100 to 0700) followed by a gradual decrease to low levels during the late light phase (1300 to 1900). Averaged across the last 5 days of $24-\mathrm{h}$ access under the discrete trial procedure, the percent of total responding that occurred in the dark phase was $47 \%( \pm 1)$ for females and $56 \%( \pm 2)$ for males. A repeated measures ANOVA on intake in the light phase $v s$ dark phase averaged across days 3-7 revealed a significant effect of sex $(\mathrm{F}(1,33)=6.7, p<0.05)$, and a significant interaction of sex and phase $(\mathrm{F}(1,33)=10.1$, $p<0.01)$, but a nonsignificant effect of phase $(p>0.05)$. Thus, after the initial 'binge', females continued to show a dysregulated pattern of use with high levels of intake occurring throughout the light/dark cycle, whereas, in males responding was more selective to the dark phase.

Despite high levels of intake under the 24-h access discrete trial procedure, animals maintained their body weights. Specifically, compared to the body weights observed prior to 24 -h access to cocaine under the discrete trial procedure, the body weights observed immediately following discrete trial cocaine self-administration were increased by an average of $1.7 \%( \pm 1.1)$ in females and decreased by an average of $1.7 \%( \pm 1.6)$ in males. A repeated measures ANOVA revealed a significant effect of group $(\mathrm{F}(1,33)=100.9, \quad p<0.0001)$, but nonsignificant effects of time $(p>0.05)$ and the interaction of time and group $(p>0.05)$. However, it should be noted that selfadministration under these high access conditions resulted in signs of severe toxicity, and these signs were apparent in females. Specifically, of 20 females tested under these high access conditions, three females died or showed rapid weight loss over the course of the 7 days 24 -h access discrete trial procedure and one female showed signs of toxicity during the initial withdrawal period. In males, however, little evidence of toxicity was observed among the 18 animals tested in the present study or in previous studies with males tested under similar conditions (Roberts et al, 2002).
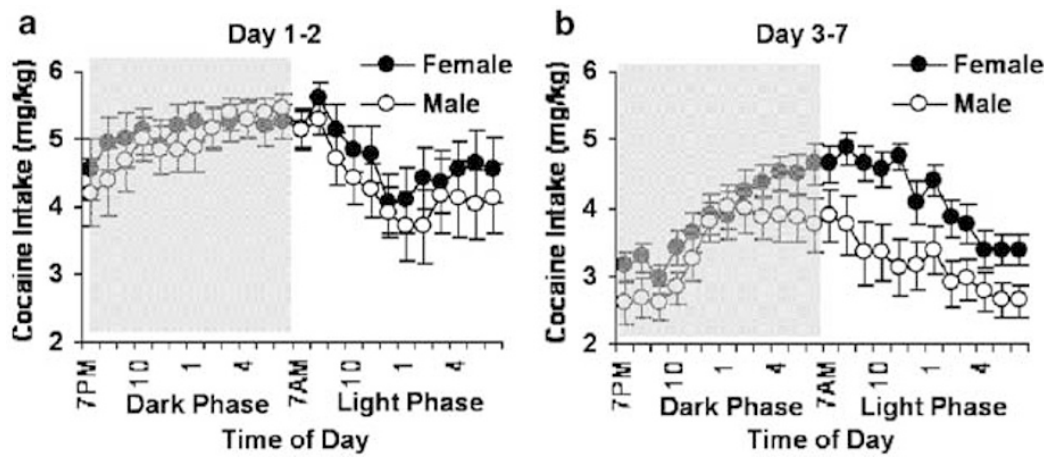

Figure I Sex differences in diurnal control over cocaine self-administration behavior under a 24-h access discrete trial procedure (a) Mean ( \pm SE) hourly intake across the light/dark cycle averaged across days I-2 under the discrete trial procedure for male $(n=18)$ and female rats $(n=17)$. (b) Mean $( \pm$ SE) hourly intake across the light/dark cycle averaged across days 3-7 under the discrete trial procedure. 

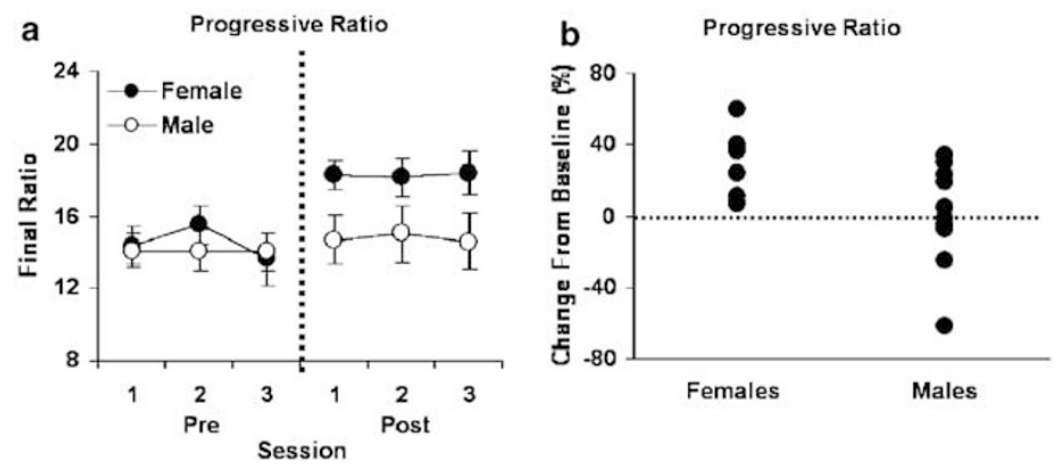

Figure 2 Sex differences in motivation to use cocaine following an extended drug-free period. (a) Final ratios reached under the progressive-ratio schedule before access to cocaine under the discrete trial procedure and after a l0-day drug-free period in male $(n=12)$ and female $(n=8)$ rats. $(b)$ Percent change in number of infusions obtained under the progressive-ratio schedule at retest from the initial baseline testing for individual male and female rats.

\section{Progressive-Ratio Responding Following the 24-h Access Discrete Trial Procedure}

Figure 2a shows data for the number of infusions obtained and the corresponding final ratios reached under the progressive-ratio schedule for male and female rats at baseline and after a 10-day forced abstinence period. We observed sex differences in progressive-ratio responding following 24-h/day access to cocaine under the discrete trial procedure. Specifically, following 10 days of forced abstinence, final ratios reached under the progressive-ratio schedule were increased from baseline levels. A repeated measures ANOVA revealed a significant main effect of time $(\mathrm{F}(1,18)=9.9, p<0.01)$, and an interaction of time and sex $(\mathrm{F}(1,18)=4.7, p<0.05)$, but a nonsignificant effect of sex $(p>0.05)$. Subsequent comparisons using separate paired $t$-tests revealed that the number of infusions obtained under the progressive-ratio at baseline compared to retest was significantly different in female $(t=4.4, \mathrm{df}=7, p<0.01)$, but not male $(p>0.05)$ rats (Figure $2 b)$. Notably, a Pearson correlation analysis revealed that there was no relationship between total intake obtained previously under the 24-h discrete trial procedure and the final ratios reached at retest indicating that the sex difference in intake cannot account for the increased breakpoints observed in females.

\section{DISCUSSION}

The goals of this investigation were to investigate sex differences in levels and patterns of intake under high cocaine access conditions using a 24 -h/day discrete trial (4, $10 \mathrm{~min}$ trials $/ \mathrm{h}$ ) procedure. A second goal was to determine whether there were sex differences in motivation to use cocaine after such access. Under these high cocaine access conditions, we observed sex differences in both patterns and levels of cocaine self-administration and in the effects of cocaine self-administration on subsequent responding for cocaine. Specifically, females self-administrated more cocaine, for longer initial periods of time, and showed a greater level of diurnal dysregulation compared to males. Moreover, following a 10-day forced abstinence period, females, but not males, showed an increase from baseline in the final ratios reached under the progressive-ratio schedule. Interestingly, no sex differences were observed for rates of acquisition as measured under the fixed-ratio 1 schedule or in motivation to self-administer cocaine as measured by progressive-ratio responding. These findings suggest that prior to 24 -h access to cocaine under the discrete trial procedure, the cocaine dose tested $(1.5 \mathrm{mg} / \mathrm{kg})$ maintained similar levels of responding in males and females. Taken together, these findings suggest that sex differences are revealed under high cocaine access conditions and that such access leads to sex-specific changes in motivation to obtain cocaine.

A great deal of attention has been paid to factors that predict a vulnerability to initiate cocaine self-administration under low access conditions, and a broad range of factors have been identified including sex and hormonal influences (Carroll et al, 2002; Campbell et al, 2002; Lynch et al, 2001; Lynch and Carroll, 1999). Few studies, however, have investigated vulnerability factors under extended access conditions. In this study, total levels of intake, initial 'binge' length, and patterns of responding across the light/dark cycle were compared between males and females in an effort to quantify diurnal control over cocaine self-administration behavior. Patterns observed in male rats were similar to those reported previously under similar testing conditions (Fitch and Roberts, 1993; Roberts et al, 2002; Morgan et al, 2002; Lynch and Roberts, 2004). Specifically, the present results showed that males took every infusion available for the first $28 \mathrm{~h}$ under the discrete trial procedure and that during this time responding occurred throughout the light/ dark cycle. After the initial 'binge', intake decreased and responding became more selective to the dark cycle. Although a similar pattern of intake was also observed in females, we found that compared to males, females 'binged' for a longer initial period of time taking every infusion available for an average of $39 \mathrm{~h}$, self-administered more cocaine over the 7 days of discrete trial access, and showed a prolonged disruption in the diurnal control over responding with high levels of intake occurring in the light and dark phases. Thus, the present findings extend previous findings of sex differences under low access conditions to include self-administration under high access conditions.

Cocaine self-administration under the discrete trial procedure followed by a 10-day forced abstinence period produced a significant increase in the motivation to self- 
administer cocaine in female rats as measure by progressive-ratio responding. These effects may indicate a sex difference in the development of sensitization to the reinforcing effects of cocaine, a phenomenon that is believed to occur following repeated, intermittent exposure that may underlie the development of addiction and serve to perpetuate the addiction cycle (Robinson and Berridge, 2001). In the present experiment, we tested males and females using only one dose of cocaine; thus, it is difficult to conclude whether or not the increased progressive-ratio responding reflects the development of sensitization to the reinforcing effects of cocaine. However, these data are consistent with the larger body of literature describing sex differences in the development of sensitization to the locomotor activating and reinforcing effects of psychostimulants. For example, previous research has shown that female rats display a greater locomotor response and more robust behavioral sensitization and that these effects occur following lower doses of drugs (Glick and Hinds, 1984; Hu and Becker, 2003; Haney et al, 1994; Sell et al, 2000). These findings are also consistent with preclinical studies showing that female rats acquire cocaine self-administration at a faster rate (Lynch and Carroll, 1999; $\mathrm{Hu}$ et al, 2004) and respond at higher levels under 'relapse' or reinstatement testing conditions (Lynch and Carroll, 2000) and with clinical reports showing that during abstinence women report higher levels of cocaine craving than do men (Robbins et al, 1999). It should be noted, however, that the present findings are probably not due to simply a sex difference in total intake during the 24-h access discrete trial testing in that there was no correlation between total intake and change in progressive-ratio responding. Previous work with male animals has also revealed that sensitization can develop to drug-associated cues (ie Grimm et al, 2001), but the contribution of primary $v s$ secondary drug cues has yet to be explored in female rats. Further research is necessary to determine possible mechanisms that underlie these sex differences, and to examine conditions that induce sensitization (eg cue- $v s$ drug-induced reinstatement) in male and female rats.

Notably, no sex differences were observed under the fixed-ratio schedule or the progressive-ratio schedule prior to 24 -h access to cocaine under the discrete trial procedure. These findings are in contrast to our previous work (Lynch and Carroll, 1999; Carroll et al, 2002) and the work of others (Hu et al, 2004; Roberts et al, 1989) showing that female rats acquire cocaine self-administration at a faster rate and respond at higher levels under the progressive-ratio schedule than do males. A likely explanation for this discrepancy is that the high cocaine dose tested in the present experiment may have obscured sex differences. Specifically, when males and females are compared under conditions that promote slow and low rates of acquisition (eg low doses, food satiated), rates of acquisition are highly variable and sex differences are revealed with females acquiring at a much faster rate than males. However, under conditions that promote rapid rates of acquisition (eg high doses, operant pretraining, food deprivation), like those used in the present experiment, males and females do not differ in rates of acquisition (Haney et al, 1995; Roberts et al, 1989; Lynch et al, 2001). Interestingly, under the 24-h discrete trial procedure this same dose of cocaine main- tained differential levels of responding in males and females. These findings suggest that although the dose of cocaine tested in the present experiment is considered to be relatively high under fixed- and progressive-ratio testing conditions, it may be at a threshold level for the production of sustained high levels of intake for male, but not female rats. In fact, the patterns and levels of intake observed in female rats in the present experiment are similar to those observed in male rats tested under higher cocaine access conditions (eg 5 trials/h with $1.5 \mathrm{mg} / \mathrm{kg} / \mathrm{inj}$. cocaine; Lynch and Roberts, 2004; Roberts et al, 2002). The idea that lower doses are required to produce sustained levels of intake in females compared to males is consistent with previous research showing that in females lower cocaine doses are necessary to induce stereotypy (Walker et al, 2001), and relapse responding (Lynch and Carroll, 2000).

Although 24-h discrete trial cocaine self-administration did not seem to impact body weights in females or males, signs of severe toxicity and death were apparent in some female rats. In humans, there is evidence to suggest that cocaine use may impose differential consequences on the health of women and men. For example, in a study comparing crack addicts, Dudish and Hatsukami (1996) reported that more women than men had emergency room visits following crack use. However, there is also evidence to suggest that females are less vulnerable than males to some toxic effects of cocaine. For example, higher doses of acutely administered cocaine are required to produce cardiovascular toxicity in female compared to male rats (Morishima et al, 1993). Future research is necessary to further investigate the mechanisms that underlie sex differences in toxicity following chronic, high levels of use.

In order to understand the neurobiology of addiction, it is critical to investigate animal models that capture the elements believed to characterize human cocaine addiction, namely compulsive patterns of use and a propensity to relapse (American Psychiatric Association, 1994). Here, these characteristics were demonstrated to be greater in females compared with males, suggesting that the 24-h discrete trial self-administration procedure may be a useful model of addiction. Notably, these findings were observed despite evidence for similar pharmacokinetic properties of cocaine in male and female rats (Bowman et al, 1999). Rates of drug addiction are higher in men, though rates are increasing in women (Substance Abuse and Mental Health Services Administration, 2001). There is some evidence to suggest that sex differences in prevalence of use may be due to increased drug use opportunity in men compared to women (Van Etten et al, 1999). Our results suggest that women may have a greater biological vulnerability to become addicted to drugs, and that once addicted, may be more motivated than men to continue to use drugs. Although the mechanisms underlying these sex differences are not clear, ovarian steroids, and estrogen in particular, are probable candidates as previous work has shown that estrogen plays a critical role in modulating the behavioral and reinforcing effects of cocaine in both women (Sofuoglu et al, 1999) and female animals (Hu and Becker, 2003; Lynch et al, 2000, 2001; Roberts et al, 1989; Sircar and Kim, 1999). Current research is underway to investigate the effects of ovarian hormones on patterns of cocaine self-administration under high access conditions. 


\section{ACKNOWLEDGEMENTS}

We are grateful to Victoria Stewart for her technical assistance, and to Drs Marilyn Carroll, Yavin Shaham, Theresa Lee, and Barry Everitt for their critical comments on a previous version of this paper. We acknowledge the Yale Interdisciplinary Women's Health Research Scholar Program on Women and Drug Abuse, NIH Office of Research on Women's Health. This work was supported by NIDA grants DA11717 and DA016556 (JRT) and DA114038 (WJL).

\section{REFERENCES}

Ahmed SH, Koob GF (1998). Transition from moderate to excessive drug intake: change in hedonic set point. Science 282: 298-300.

Ahmed SH, Koob GF (1999). Long-lasting increase in the set point for cocaine self-administration after escalation in rats. Psychopharmacology 146: 303-312.

American Psychiatric Association Diagnostic and Statistical Manual of Mental Disorders, 4th edn. American Psychiatric Press: Washington, DC; 1994.

Arnold JM, Roberts DCS (1997). A critique of fixed and progressive ratio schedules used to examine the neural substrates of drug reinforcement. Pharmacol Biochem Behav 57: 441-447.

Bowman BP, Vaughan SR, Walker QD, Davis SL, Little PJ, Scheffler, $\mathrm{NM}$ et al (1999). Effects of sex and gonadectomy on cocaine metabolism in the rat. J Pharmacol Exp Ther 290: 1316-1323.

Brady KT, Randall CL (1999). Gender differences in substance use disorders. Psychiatr Clin North Am 22: 241-252.

Campbell UC, Morgan AD, Carroll ME (2002). Sex differences in the effect of baclofen on the acquisition of cociane selfadministration in rats. Drug Alcohol Depend 66: 61-69.

Carroll ME, Morgan AD, Campbell UC, Lynch WJ, Dess NK (2002). Influence of estrogen in the acquisition of intravenously selfadministration in rats selectively bred for differential saccharin intake: phenotype and sex differences. Psychopharmacology 161: 304-313.

Dudish SA, Hatsukami DK (1996). Gender differences in crack users who are research volunteers. Drug Alcohol Depend 42: 55-63.

Elman I, Karlsgodt KH, Gastfriend DR (2001). Gender differences in cocaine craving among non-treatment-seeking individuals with cocaine dependence. Am J Drug Alcohol Abuse 27: 193-202.

Evans SM, Haney M, Fischman MW, Foltin RW (1999). Limited sex differences in response to 'binge' smoked cocaine use in humans. Neuropsychopharmacology 21: 445-454.

Fitch TE, Roberts DCS (1993). The effects of dose and access restrictions on the periodicity of cocaine self-administration in the rat. Drug Alcohol Depend 33: 119-128.

Freeman WM, Brebner K, Patel KM, Lynch WJ, Roberts DC, Vrana KE (2002). Repeated cocaine self-administration causes multiple changes in rat frontal cortex gene expression. Neurochem Res $1181-1192$.

Gawin FH (1991). Cocaine addiction: psychology and neurophysiology. Science 2251: 1580-1586.

Glick SD, Hinds PA (1984). Sex differences in sensitization to cocaine-induced rotation. Eur J Pharmacol 99: 119-121.

Grimm JW, Hope BT, Wise RA, Shaham Y (2001). Neuroadaptation. Incubation of cocaine craving after withdrawal. Nature 412: 141-142.

Haney M, Castanon N, Cador M, Le Moal M, Mormede P (1994). Cocaine sensitivity in roman high and low avoidance rats is modulated by sex and gonadal hormone status. Brain Res 645: 179-185.
Haney M, Maccari S, Le Moal M, Simon H, Piazza PV (1995). Social stress increases the acquisition of cocaine self-administration in male and female rats. Brain Res 698: 46-52.

$\mathrm{Hu}$ M, Becker JB (2003). Effects of sex and estrogen on behavioral sensitization to cocaine in rats. J Neurosci 23: 693-699.

Hu M, Crombag HS, Robinson TE, Becker JB (2004). Biological basis of sex differences in the propensity to self-administer cocaine. Neuropsychopharmacology 29: 81-85.

Jentsch JD, Taylor JR (1999). Impulsivity resulting from frontostriatal dysfunction in drug abuse: implications for the control of behavior by reward-related stimuli. Psychopharmacology 146: 373-390.

Koob GF (1992). Neural mechanisms of drug reinforcement. Ann NY Acad Sci 28: 171-191.

Kosten TR, Kosten TA, McDougle CJ, Hameedi FA, McCance EF et al (1996). Gender differences in response to intranasal cocaine administration to humans. Biol Psychiatry 39: 147-148.

Lynch WJ, Arizzi MN, Carroll ME (2000). Effects of sex and the estrous cycle on regulation of intravenously self-administered cocaine in rats. Psychopharmacology 152: 132-139.

Lynch WJ, Carroll ME (1999). Sex differences in the acquisition of intravenously self-administered cocaine and heroin in rats. Psychopharmacology 144: 77-82.

Lynch WJ, Carroll ME (2000). Reinstatement of cocaine selfadministration in rats: sex differences. Psychopharmacology 148: 196-200.

Lynch WJ, Roberts DCS (2004). The effects of cocaine selfadministration on food-reinforced responding using a discrete trial procedure. Neuropsychopharmacology, (in press).

Lynch WJ, Roth ME, Carroll ME (2002). Biological basis of sex differences in drug abuse: preclinical and clinical studies. Psychopharmacology 164: 121-137.

Lynch WJ, Roth ME, Mickelberg JL, Carroll ME (2001). Role of estrogen in the acquisition of intravenously self-administered cocaine in female rats. Pharmacol Biochem Behav 68: 641-646.

Mello NK, Mendelson JH (2002). Cocaine, hormones, and behavior: clinical and preclinical studies. Horm Brain Behav 5: 665-745.

Morgan D, Brebner K, Lynch WJ, Roberts DCS (2002). Increases in the reinforcing efficacy of cocaine after particular histories of reinforcement. Behav Pharmacol 13: 389-396.

Morishima HO, Abe Y, Matsuo M, Akiba K, Masaoka T, Cooper TB (1993). Gender-related differences in cocaine toxicity in the rat. J Lab Clin Med 122: 157-163.

Robbins SJ, Ehrman RN, Childress AR, O’Brien CP (1999). Comparing levels of cocaine cue reactivity in male and female outpatients. Drug Alcohol Depend 53: 223-230.

Roberts DCS, Bennett SAL, Vickers GJ (1989). The estrous cycle affects cocaine self-administration on a progressive ratio schedule in rats. Psychopharmacology 98: 408-411.

Roberts DCS, Brebner K, Vincler M, Lynch WJ (2002). Patterns of cocaine self-administration in rats produced by various access conditions under a discrete trial procedure. Drug Alcohol Depend 67: 291-299.

Robinson TE, Berridge KC (2001). Incentive-sensitization and addiction. Addiction 96: 103-114.

Sell SL, Scalzitti JM, Thomas ML, Cunningham KA (2000). Influence of ovarian hormones and estrous cycle on the behavioral response to cocaine in female rats. J Pharmacol Exp Ther 293: 879-886.

Sircar R, Kim D (1999). Female gonadal hormones differentially modulate cocaine-induced behavioral sensitization in Fischer, Lewis, and Spraque-Dawley rats. J Pharmacol Exp Ther 289: 54-65.

Sofuoglu M, Dudish-Poulsen S, Nelson D, Pentel PR, Hatsukami DK (1999). Sex and menstrual cycle differences in the subjective effects from smoked cocaine in humans. Exp Clin Psychopharmacol 7: 274-283. 
Sex differences in 24-h access to cocaine

WJ Lynch and JR Taylor

Substance Abuse and Mental Health Services Administration (2001). Summary of Findings from the 2000 National Household Survey on Drug Abuse. Office of Applied Studies, NHSDA Series H-13, DHHS Publication No. (SMA) 01-3549, Rockville, MD.

Tornatzky W, Miczek KA (2000). Cocaine self-administration 'binges': transition from behavioral and autonomic regulation toward homeostatic dysregulation in rats. Psychopharmacology 148: 289-291.
Van Etten ML, Neumark YD, Anthony JC (1999). Male-female differences in the earliest stages of drug involvement. Addiction 94: 1413-1419.

Walker QD, Cabassa J, Kaplan KA, Li ST, Haroon J, Spohr HA et al (2001). Sex differences in cocaine-stimulated motor behavior: disparate effects of gonadectomy. Neuropsychopharmacology 25: $118-130$. 